\title{
Simuladores Degenerados: Una lectura foucaultiana de "La Simulación de la locura" de José Ingenieros
}

Sandra Caponi'

\section{Resumen}

Este artículo discute el problema que constituye el eje articulador del estudio que José Ingenieros dedica a la simulación de la locura: los delincuentes simuladores. Se trata de sujetos que han cometido un delito y que, con el objetivo de liberarse de la prisión, simulan haber actuado sin conciencia de sus actos. Por la mediación de la figura de los simuladores, Ingenieros ilustra el momento preciso en el que se inicia, en la psiquiatría Argentina, la construcción de ese continuum psiquiátrico-criminológico que permite abordar en términos médicos las cuestiones jurídicas más variadas. A partir de ese momento será posible, por la mediación de la teoría de la degeneración, que un conjunto ambiguo de comportamientos, ingrese al campo de la observación psiquiátrica. Para Ingenieros, solo cuando el derecho integre esa nueva perspectiva psiquiátrica, el problema de la simulación podrá finalmente resolverse.

Palabras clave: Ingenieros. Simulación. Psiquiatría. Degeneración. Derecho. José Ingenieros and degeneration in simulated madness

L'article 64, monsieur Maigret! N'oubliez pas l'article 64! Pourquoi cet avocat d'affaires, qu'on venait consulter de partout, s'hipnotisait-il ainsi sur le seul article du Code à traiter de la responsabilié humaine? Prudemment. Sans donner la moindre definition de la démence. Et en la limitan au momento de l'action, c'est-à-dire au momento du crime.

Georges Simenon, Maigret hésite.

1 Professora Titular do Departamento de Sociologia e Ciência Política da Universidade Federal de Santa Catarina. Doutora em Filosofia pela Unicamp e Pós-doutorado em Paris VII. Pesquisadora de CNPq.

E-mail: sandracaponi@gmail.com. 
Insidiosamente, lentamente, de forma reptante y segmentada se organiza una penalidad centrada en lo que se es: han sido necesarios más de cien años para que esta noción de individuo peligroso, que estaba virtualmente presente en la monomanía de los primeros alienistas, fuese aceptada por el pensamiento jurídico. Al cabo de cien años esta noción se ha convertido en un tema central de los exámenes periciales psiquiátricos.

Michel Foucault, 1981.

\section{Introducción}

Pretendo analizar aquí la temática que constituye el eje articulador del libro La simulación de la locura publicado, en 1903, por el psiquiatra argentino José Ingenieros: los delincuentes simuladores Los estudios que Michel Foucault dedica a la intersección locura-criminalidad, particularmente aquellos dedicados a la construcción de la noción de "individuo peligroso, servirán como marco de análisis para abordar este libro. Este texto de Ingenieros, surge inicialmente como resultado de la tesis que defiende en el año 1900 para obtener el título de médico. Tres años más tarde, esa tesis será publicada en forma de libro, con un capítulo inicial denominado La simulación en la lucha por la vida. Por esta obra, la Academia de Medicina de Buenos Aires le otorgará a Ingenieros la medalla de oro destinada a premiar la mejor obra científica argentina. De modo que, como afirma Cristina Fernandez (2006), La simulación de la locura, cimentará la fama de Ingenieros como el más prestigioso alienista, sociólogo y criminalista argentino de su época²

Aunque Ingenieros habla de una infinita variedad de tipos de simulación, su estudio se centra en tres formas privilegiadas: los que simulan una enfermedad para eludir el servicio militar, los que fingen para aprovecharse de la beneficencia y por fin, los simuladores de la locura. A este último grupo pertenecen los delincuentes simuladores a los que se refiere este escrito.

$\mathrm{Al}$ leer el texto de Ingenieros surge inevitablemente una pregunta que Andrew Lakoff (2014, p. 848) formulá de este modo: "Why would so many

2 Sobre la influencia de los estudios de José Ingenieros en el desarrollo del positivismo argentino ver, entre otros: Vezzetti (1988), Biagini (1980), Vermeren y Villavicencio (1998), Falcone (2012), Stagnano (2006), Galfiore (20/2). 
readers have been intereseted in this catalogue of the varieties of ways that madness was linked to simulacion??"Sin embargo, los estudios dedicados a ese libro, lejos de considerarlo como un catálogo de formas de simulación, generalmente se detuvieron a analizar la definición que Ingenieros presenta como eje articulador de su estudio: "La simulación es un hecho fraudulento en la lucha por la vida” (INGENIEROS, 1962a, p. 107).

A partir de allí han proliferado estudios dedicados a analizar el evolucionismo biológico de Ingenieros, sus referencias a la simulación en la "lucha por la vida" y el papel de "la adaptación al medio", discutiendo si esas ideas provenían de una filiación teórica darwiniana, spenceriana o neo-lamarkiana. Ese debate aquí será dejado de lado. Pues, el hecho de que existan delincuentes que simulan haber actuado sin tener conciencia de sus actos, para liberarse de la cárcel, nada tiene que ver con las formas de mimetismo, mulleriano o batesiano, que eran consideradas y aceptadas por los evolucionistas de fines del Siglo XIX e inicios de siglo XX, como formas paradigmáticas de entender la simulación adaptativa ${ }^{3}$.

Por otra parte, el problema representado por la locura simulada, ya estaba presente en autores como Pinel, mucho antes de que se hablara de lucha por la vida. De modo que, para entender el problema de los delincuentes simuladores, que quieren eludir una condena, no parece ser necesaria ninguna referencia al evolucionismo, aunque Ingenieros presente sus argumentos utilizando una retórica darwiniana ${ }^{4}$.

Otros estudios dedicados a La Simulación de la locura, se han detenido también a discutir la relación locura, crimen y peligrosidad, vinculándola con la preocupación por crear las bases de la nación argentina, deteniéndose a analizar la simulación desde la perspectiva abierta por los debates sobre la cuestión social y "la mala vida". Estableciendo, muchas veces, conexiones entre las estrategias de construcción del estado argentino y la temática del

3 Gayon, J. Darwin et l'après Darwin. Une histoire de I'hipothèse de sélection naturelle. Paris, Ed Kimé, 1992, p. 198; Crpenter, G EFord, E. Mimicry. London, ED. MatheeuenEco, 1033, p. 12.

4 Para Adrià Casinos, refiriéndose al fin del XIX: "La "lucha por la vida" era una forma de hablar, una frase hecha, utilizada en aquel momento en todos los núcleos positivistas, tanto del Viejo como del Nuevo Mundo". Casinos, A. Un evolucionista en El Plata: Florentino Ameghino. Buenos Aires, Ed. Fundación de História Natural Felix de Azara. 2012, p. 98.

5 Terán, O. "José Ingenieros: pensar la nación", Buenos Aires, Alianza, 1986. 
evolucionismo social. No pretendo retomar aquí, una vez más, esos debates, sino intentar entender el suelo epistemológico que, específicamente en el campo de la psiquiatría, pudo servirle a Ingenieros de auxilio para discutir el problema de la simulación de la locura.

La Simulación de la locura, como otros textos del mismo autor, se caracteriza por cierto alarde de erudición. Vemos desfilar allí, las más variadas referencias a biólogos, criminólogos, juristas, psiquiatras, pero también abundan referencias literarias como menciones a Ulises o Hamlet. Frente a esa proliferación de autores no resulta fácil entender cuáles eran las filiaciones epistemológicas de Ingenieros, particularmente en lo que se refiere a la medicina mental. Aunque existen menciones a diferentes escuelas, parece haber un modelo explicativo que se repite, tanto en las argumentaciones teóricas como en los casos presentados. Repetidamente aparecen explicaciones referidas a las nociones articuladoras de la teoría de la degeneración, como anomalías, locuras menores, estigmas físicos y morales o herencia mórbida, pretendo analizar el papel que ocupa esa teoría en la construcción de los argumentos de Ingenieros sobre la simulación de la locura por los delincuentes.

En las últimas décadas del siglo XIX, la psiquiatría estaba fuertemente influenciada por esa nueva representación de las patologías que habla de degeneraciones hereditarias. Surgen entonces diversos estudios preocupados por aclarar y descubrir nuevos síndromes de degeneración, nuevos estigmas físicos y psíquicos. Ian Hacking (2000) dirá que en ese momento se inicia un verdadero 'programa de investigación', muy fructífero y capaz de concentrar los esfuerzos de todos aquellos que compartían una misma preocupación: la de ampliar el alcance y los límites de la medicina mental. Aparece así un amplio espectro de síndromes de degeneración que incluye las manías de persecución, las aberraciones sexuales, las anomalías de comportamiento y una inmensa variedad de miedos y temores. Este programa de investigación no solo tendrá impacto en Francia, Italia y Alemania, sino también en América Latina.

El texto de Ingenieros parece poner en evidencia la influencia de esa teoría en Argentina, en este caso concreto, para pensar de otro modo un viejo problema que obcecaba a los psiquiatras: la simulación de la locura. Lakoff (2014) se refiere a un artículo publicado por Ingenieros en el ańo 1902, "Responsabilidad penal de los degenerados impulsivos", en coautoría 
con Ramos Mejía y Solari ${ }^{6}$, mostrando la relevancia dada a los estigmas de degeneración. Sin embargo, y aunque también existan inúmeras referencias de Ingenieros a la teoría de la degeneración en La Simulación de la locura, fundamentalmente cuando analizamos los casos presentados, existen pocos estudios dedicados a tematizar las huellas dejadas por esa teoría en el análisis que Ingenieros dedica al problema de la simulación.

\section{Psiquiatría y simulación}

Aunque el problema de la simulación afecta a juristas y criminólogos, cabe a la psiquiatría decidir si el delincuente es efectivamente un alienado o si se trata de un simple caso de simulación. En ese acto, en ese procedimiento de arbitraje entre la verdad y la falsedad de los síntomas se juegan inúmeras cuestiones jurídicas, criminológicas, psiquiátricas y también epistemológicas. Ocurre que, como afirma Foucault:

El gran problema de la historia de la psiquiatría en el siglo XIX no es un problema de conceptos, no es en absoluto el problema de tal o cual enfermedad: el verdadero problema, la cruz de la psiquiatría decimonónica, no es la monomanía y ni siquiera la histeria. Si se admite que en el poder psiquiátrico la cuestión de la verdad jamás se plantea, puede entenderse que la cruz de la psiquiatría decimonónica sea simplemente el problema de la simulación. (2003, p. 160).

El problema de la simulación de la locura parece ser contemporáneo al nacimiento de la psiquiatría moderna. Vemos aparecer este problema en los principales textos de la historia de la psiquiatría a lo largo del siglo XIX e inicios de siglo XX. Inicialmente surge como tema de interés en el Tratado de Pinel, particularmente en el capítulo dedicado a la Manía simulada y medios de reconocerla (PINEL, 1908, p. 271). Más tarde, a mediados del siglo XIX, este será un tema de preocupación que concentra el interés de los degeneracionistas. Benedict August Morel (1857), analiza un caso particular de simulación, el caso Desrosieres, publicado en los Anales Médico-psicológicos. El psiquiatra Armand Laurent (1866) escribe Étude médico-légale sur la simulation de la folie. Luego con Charcot (1887), a propósito de la histeria, la cuestión de la

6 Ingenieros, Ramos Mejía y Solari 1902, "Responsabilidad penal de los degenerados impulsivos". 
simulación vuelve a escena, por ejemplo en Leçons sur les maladies du système nerveux, faites a la Salpêtrière.

Foucault afirma que existe un momento de ruptura en el sistema médico jurídico, "un momento que fue particularmente fecundo para las relaciones sostenidas entre la psiquiatría y el derecho penal: los últimos ańos del siglo XIX y los primeros años del siglo XX: la etapa que va entre el Primer Congreso de Antropología Criminal, en 1885, a la publicación por Prins de La Défense Sociale, en 1910" (FOUCAULT, 1981, p. 414). Fue justamente en ese período que José Ingenieros elaboró su estudio sobre la simulación.

En ese momento se establecieron nuevas alianzas entre el discurso médico y el discurso jurídico. Una transformación significativa posibilitada por el progresivo abandono de la noción de monomanía homicida, que había servido como recurso explicativo para todo impulso violento o criminal. Esa noción psiquiátrica y jurídica, totalmente centrada en el acto criminal, excluía cualquier pregunta que no se dirigiera al homicidio consumado. Esto es, cerraba las puertas para la posibilidad de interrogarse, ya no por el crimen, sino por el sujeto criminal. Foucault afirma que en ese período:

La monomanía será abandonada porque la idea de las enfermedades mentales de evolución compleja ya se había abierto camino: la idea de enfermedades mentales que pueden presentar tal o cual síntoma específico en tal o cual estadio de su desarrollo, no solo en el plano individual sino en el de las generaciones sucesivas: es la idea de degeneración. (2014, p. 237).

Es en ese marco que pretendo situar las reflexiones que Ingenieros ${ }^{8}$ dedica al problema de la simulación. La idea de impulso violento y criminal asociada a falta de conciencia, será poco a poco desplazada. Se dirá que un alienado puede conservar su capacidad de razonar y poseer, aun así, impulsos incontrolables que lo lleven a cometer actos violentos. Esto significa que la simulación de la locura con el objetivo de evitar una condena no sería una estrategia exclusivamente empleada por sujetos normales y conscientes, sino que también podría ser utilizada por los alienados. Para Ingenieros:

7 Ver al respecto: Laurent (1866); Garbini (1906), Morel (1857), Lakoff (2005).

8 Sobre la influencia de los estudios de José Ingenieros en el desarrollo del positivismo argentino ver, entre otros: Biagini (1980), Vermeren y Villavicencio (1998), Falcone (2012). Stagnano (2006), Fernandes (2006). Ver también, Oscar Terán (1987) y Hugo Vezzetti (1988). Ambos se detienen a analizar ese momento histórico puntual en Argentina de finales del siglo XIX e inicios del siglo XX. De acuerdo a Oscar Terán (1987, p. 14): "En el área del ensayo positivista argentino, lo más significativo transcurre centralmente en las obras de José Ramos Mejía, Agustín Álvarez, Carlos Octavio Bunge y José Ingenieros". 
Si la locura fuera, en un sentido simple y absoluto, una pérdida de la posibilidad de razonar, como generalmente suele creerse, parecería absurdo suponer en el alienado la capacidad de apelar a la simulación para obtener ventajas, de cualquier índole, en la lucha por la vida. y parecería aún más absurdo que, en ciertos casos, el alienado intentara simular síntomas de alienación mental distintos de los verdaderos. Pero la creencia de que los alienados son incapaces de razonar ha sido ya desterrada entre los alienistas, y aun de entre las personas cultas, conocedoras de algunos elementos de psicopatología. (INGENIEROS, 1962a, p. 135).

Es preciso, entonces, pensar a las enfermedades mentales como un continuo que va de las pequeñas anormalidades cotidianas a las formas más graves del delito. De modo que a cada estadio de desarrollo de una enfermedad psiquiátrica se podrán atribuir síntomas específicos que se suceden con gravedad creciente. En este continuum psiquiátrico- criminológico no solo los grandes crímenes, sino cualquier infracción, podrán pasar a ser objeto de intervención psiquiátrica. En esa línea de continuidad entre lo normal y lo patológico aparecen esas pequeñas anomalías que, en las últimas décadas del siglo XIX, pasaron a tener estatuto de enfermedad mental. Es por eso que Foucault, al referirse a los teóricos de la degeneración dirá: "en definitiva, entre una mujer que roba ropa interior en una tienda y una madre que cocina el muslo de su hija en una olla, tanto en un caso como en otro, se puede y se debe hacer la misma pregunta: no hay locura en esto?” (FOUCAULT 2014, p. 238).

Esa continuidad entre lo normal y lo patológicos supone la idea de una variedad de manifestaciones patológicas, cuya gravedad aumentaría lo largo de la vida de un individuo, desde la infancia a la edad adulta, y a lo largo de las sucesivas generaciones de una misma familia. De modo que la psiquiatra puede intervenir en cualquier situación que represente una amenaza, presente o futura, para la sociedad. En ese marco si sitúa Ingenieros cuando afirma:

La última mitad del siglo XIX vio florecer curiosos e interesantes estudios de psicopatología no sospechados por los clínicos de antaño. Junto al hombre normal y al loco, anastomosándose con ambos, se describieron tipos desequilibrados, fluctuando desde el genio hasta la delincuencia, desde la mentira hasta la inversión sexual. En realidad, todos los individuos que llama Venturi “característicos” (INGENIEROS, 1962a, p. 174).

La línea de continuidad que se establece entre las pequeñas faltas y el delito monstruoso, fue posibilitada por la aceptación, difusión y expansión de la teoría de la degeneración. Una teoría que parece haber operado como eje articulador de la discusión realizada por Ingenieros sobre la simulación de la locura. 
Autores como Magnan, Sergi, Janet, Legrain, Ballet, estudiaron esos sujetos desviados del tipo medio por la neuropatía o la degeneración, sin adaptarse a los moldes clínicos de los tratadistas clásicos. Ellos revelan la mediocridad de la vieja clínica, cuyos esquemas desvencijados no abarcan los casos, para ella inexplicables, que saltan a la vista del psicólogo concienzudo que contempla la infinita variedad de las anomalías. Junto a esas anormalidades permanentes, inconfundibles con la locura, no obstante lindar con ella, encontramos innumerables trastornos transitorios de la psiquis. (INGENIEROS, 1962a, p. 175).

Ingenieros ilustra de ese modo, el momento en que se inicia, en la psiquiatría Argentina, la construcción de ese "continuum psiquiátrico y criminológico que permite abordar en términos médicos cualquier grado de la escala penal" (FOUCAULT, 2014, p. 238).

Aunque la teoría de la degeneración fue enunciada por primera vez por Morel $^{9}$ (FOUCAULT, 1999; HUERTAS, 1987; COFFIN, 2003; CAPONI, 2015), será con Magnan (1898) que comenzarán a establecerse las articulaciones entre el discurso psiquiátrico y la medicina legal. Como afirma Truelle: "[...] en la época de Magnan las pericias médico-legales eran sino excepcionales, al menos raras. Él contribuyó ampliamente a extender y generalizar la práctica psiquiátrica en los tribunales" (1935, p. 770).

Truelle (1935) dirá que fue justamente Magnan quien ayudó generalizar la práctica de la medicina mental en los tribunales. Magnan insistirá en la necesidad de realizar exámenes psiquiátricos para identificar simuladores y evitar que los alienados sufran una condena inmerecida. Postula también la existencia de una categoría amplia de delincuentes que, aunque no deberían ser internados en asilos psiquiátricos, tampoco podían ser considerados como individuos normales. $\mathrm{Y}$ es en ese campo abierto por los teóricos de la degeneración ${ }^{10}$, conjuntamente con los desarrollos de la antropología positiva, que Ingenieros se sitúa para responder al problema de la simulación. Dirá que:

Es necesario sobreponerse a las disidencias entre las escuelas francesa, italiana y alemana que, en detalle, dan de la degeneración distintas interpretaciones, aunque coincidiendo en lo fundamental, siguiendo las huellas luminosamente trazadas de Morel a Magnan.

9 Morel, tratado de degeneración

10 Sobre la teoría de la degeneración, además de los estudios ya referenciados sugerimos la lectura de los textos clásicos de Molloy (1988) y Pick (1999). Sobre la relación entre medicina legal, higiene y eugenia ver Miranda y Vallejo (20/2). 
Para nuestro objeto basta señalar que la locura y la criminalidad están emparentadas por la degeneración. Locura y delito son dos formas antisociales de actividad individual florecidas sobre un mismo fondo de anormalidad; pueden germinar por separado, pero pueden hacerlo simultáneamente. (INGENIEROS, 1962a, p. 229).

La teoría de la degeneración permitirá situar al problema de la simulación de la locura en un nuevo espacio de análisis. Ya no se trata ni de sujetos sanos que conscientemente decidieron fingir locura para ocultar un acto criminal, ni de alienados que cometieron crímenes como reacción a impulsos irracionales incontrolables. A partir de ese momento, entre la clásica locura plagada de delirios y alucinaciones y la completa normalidad, se añade una serie de anomalías definidas como patologías mentales: los heredo-degenerados, cuya clasificación Magnan enuncia en este cuadro:

\section{Cuadro I - Synoptic arrangement of mental degeneracies Heredo-degenerates I I}

I- Idiocy, imbecility and mental debility.

2- (Unstableness) Cerebral anomalies: balance defect of the moral and intellectual faculties.

3- Hereditary episodic syndromes

(a) Doubt madness

(b) Fear of touching

(c) Onomatomania: I) agonizing search for a word, 2) irresistible impulse to repeat a word, 3) fear of using compromising words, etc.

(d) Arithmetomania

(e) Excessive love of animals: antivivisection madness

(f) Kleptomania, dipsomania, oniomania (buying mania)

(g) Gambling mania

(h) Pyromania and pyrophobia

(i) Homicidal and suicidal impulses

(j) Sexual aberrations, perversions,

(k) Agoraphobia, claustrophobia, topophobia

4- (a) Manic thinking, moral insanity (paranoia)

(b)Multiple deliria: delirium of ambition, religion, persecution, hypochondria.

(c) Systematized Delusion. Obsessive ideas

(e) Manic excitement, melancholic depression

II Valentin Magnan, Recherches sur les centres nerveux. Alcoolisme, folie des héréditaires dégénérés (Paris: Masson, 1893), 150. 
Muchas de estos síndromes de degeneración reaparecen en Ingenieros, caracterizados por estigmas físicos y morales y por herencia mórbida. En lugar de la oposición razón-locura, Ingenieros prefiere hablar de una infinita variedad de patologías psiquiátricas: desde los comportamientos sexuales desviados a la aberración criminal. El texto de Ingenieros presenta varios casos que muestran pequeños desvíos de comportamiento, pequeńas simulaciones como las del joven bohemio que decía ser artista, analizada en detalle por Andrew Lakoff (2014).

Diversos casos de simulación presentados por Ingenieros se refieren a sujetos degenerados. Así, en la IX Observación de La Simulación de la locura, se presenta el caso de un italiano, de treinta años, alcohólico y degenerado; la X Observación se refiere a un joven de 23 años, brasilero, que presenta estigmas físicos y psíquicos e degeneración; la XI Observación se refiere a un español, católico de cuarenta años que asesinó a su compañero de tareas, que presenta numerosos estigmas físicos degenerativos; la XIII Observación se refiere a un joven argentino de 35 años con antecedentes hereditarios, padre alcohólico y un tío homicida impulsivo(INGENIEROS, 1962a, p. 139 e ss.). Los casos continúan con referencias de este tipo a lo largo del libro.

Diez años más tarde, en el libro Criminología, las referencias a la degeneración permanecen. La Segunda Observación del Apéndice se refiere a un degenerado moral: un joven de 23 ańos, soltero, psiconeurópata constitucional, predominando numerosos estigmas de degenerativos mentales y físicos, con deficiencia congénita. La Cuarta Observación se refiere a un sujeto con antecedentes mórbidos, que pertenece a una familia de neurópatas degenerados. El texto continúa con el relato de numerosos casos semejantes. Es que, como afirma Ingenieros:

En realidad, todo degenerado es anómalo mental; no es posible hablar de degeneración puramente morfológica sin estigmas psíquicos, pues la psiquis no puede considerarse sino como función del organismo. En ese vasto cuadro, la locura y la criminalidad son como notas agudas en la gama de la degeneración, extremos de una serie donde se escalona una muchedumbre que sin ser honesta no es criminal, y sin ser cuerda no merece el manicomio. En todos ellos la degeneración psíquica acompaña a la morfológica. (INGENIEROS, 1962a,p. 63).

Ya no se trata, como en la psiquiatría clásica, de desvendar las estrategias de encubrimiento de los simuladores. Ahora se trata de conocer la vida, 
los estigmas, las patologías de la infancia, los antecedentes familiares, las enfermedades de la familia, si el padre es o no alcohólico. Pues, para Ingenieros el delincuente simulador es siempre, de un modo u otro, un degenerado.

\section{Sobre leyes y simulaciones}

Este nuevo modo de entender la psiquiatría, esta nueva forma de preguntarse por la locura, exige una completa transformación del Código Penal que permita ampliar el campo de lo que debe ser considerado como jurídicamente punible. Ocurre que:

La locura en la legislación penal está representada por formas clínicas definidas que confieren la irresponsabilidad; las anomalías psíquicas de los simuladores no corresponden al concepto clínico-jurídico de la locura como causa eximiente de la pena. El delincuente simulador no simula porque no tiene anomalías psíquicas verdaderas sino a pesar de tenerlas. (INGENIEROS, 1962a, p. 102).

Todo parecería indicar que el problema de los simuladores se vincula a las dificultades propias de un discurso jurídico centrado en la idea metafísica de responsabilidad. El Código Napoleónico francés, ya en 1830, incluye el famoso artículo 64 donde se definirá la inimputabilidad en casos de demencia o furor. Vale recordar el artículo 64 del antiguo Código Penal francés de 1810, en vigencia hasta el año 1992: "Il n'y a ni crime ni délit lorsque le prévenu était en état de démence au temps de l'action, ou lorsqu 'il a été contraint par una forcé à laquelle il n'a pu résister" 12 (SIMENON, 1968).

De acuerdo a las categorías jurídicas clásicas, cuando se descubre que los síntomas son fingidos, al sujeto identificado como simulador le corresponderá una pena determinada por la gravedad del crimen. Si al contrario, se descubre que la locura no es simulada sino real, será el saber psiquiátrico, quien deberá definir el tipo de tratamiento. Se podrá, por ejemplo, determinar la reclusión en un manicomio hasta que la salud sea restablecida y pueda volver a su vida normal. Cada uno de estos elementos está presente en el Código de la República Argentina, en vigencia desde 1887, redactado por Tejedor, al que se

12 "No hay ni crimen ni delito cuando el acusado haya estado en estado de demencia en el momento de la acción, o cuando este haya sido compelido por una fuerza a la que no ha podido resistir". 
refiere Ingenieros ${ }^{13}$. Considera este Código deficiente e insiste en la necesidad de reemplazarlo por una nueva legislación a la altura de los modernos Códigos europeos. Sin embargo, será solo en 1921 que el Código Penal argentino será reformado, "abandonando en términos generales, el modelo clásico" (GALFIONE, 1912, p. 22). Recordemos que de acuerdo al artículo 81, inciso primero, del Código redactado por Tejedor, queda eximido de pena:

El que ha cometido el hecho en estado de locura, sonambulismo, imbecilidad absoluta o beodez, completa o involuntaria; y generalmente, siempre que el acto haya sido resuelto y consumado en una perturbación cualquiera de los sentidos o de la inteligencia, no imputable al agente y durante el cual éste no ha tenido conciencia de dicho acto o de su criminalidad. (INGENIEROS, 1962a, p. 163).

En ese momento se crea la posibilidad de recurrir a la estrategia de simular la locura para salvarse de una condena, aunque no parezca existir ningún criterio válido para desenmascarar a los simuladores. Ante la ineficacia de los estudios estadísticos, basados en criterios metafísicos para delimitar normalidad de patología, solo parecía existir una forma de resolver el problema de la simulación: transformar la legislación penal existente ${ }^{14}$.

"La locura en la legislación penal existente está representada por formas clínicas definidas que confieren la irresponsabilidad" (INGENIEROS, 1962a, p. 102). Estas formas clínicas definidas responden a los diagnósticos clásicos de enfermedad mental, aquellos que, con algunas variaciones fueron definidos por Pinel: manía, melancolía, demencia e idiotismo. Por esa razón,

Los delincuentes que intentan eludir la represión penal simulan formas "clínico-jurídicas" de alienación y no anormalidades típicas. Las formas simuladas (que confieren irresponsabilidad) se refieren a cinco grupos de síndromes: I-maníacos, 2-depresivos, 3-delirantes, 4-episodio psicóticos y 5 -estados confuso demenciales. (INGENIEROS, 1962a, p. 102).

13 Este Código Penal entra en vigencia el $1^{\circ}$ de marzo de 1887 y estará en vigor hasta la promulgación del Código Penal de 1921 que, con numerosas alteraciones, está en vigencia hasta hoy, correspondiendo el artículo 81 del Código de 1887, al actual articulo 34, donde se mantiene el principio de inimputabilidad de los alienados.

14 Diversas propuestas de reformulación del Código Penal se sucedieron en la República Argentina, en lo que se refiere al artículo 81 mencionado por Ingenieros, será reemplazado por el articulo 34 en el Código penal de 1922. Alli leemos Art 34-10: "No es punible el que no haya podido en el momento del hecho, ya sea por insuficiencia de sus facultades, por alteraciones morbosas de las mismas o por su estado de inconsciencia, error o ignorancia de hecho no imputables, comprender la criminalidad del acto o dirigir sus acciones." 
Para Ingenieros, resolver el problema de la simulación, exige adecuar la legislación a las últimas conquistas científicas realizadas por la psiquiatría, integrando esa perspectiva a la antropología positiva.

Aunque el simulador sea capaz de evaluar y anticipar las consecuencias de su acción, sabiendo que al fingir las formas clínicas establecidas podrá evitar el encierro en la prisión, eso no significa afirmar que los simuladores delincuentes estén libres de sufrir otras anomalías psiquiátricas. Anomalías estas diferentes de aquellas definidas por el derecho penal. Para Ingenieros: "el delincuente no simula porque no tenga anomalías psíquicas verdaderas sino a pesar de tenerlas" (INGENIEROS, 1962a, p. 101). Pero, como las anomalías psíquicas padecidas por los delincuentes simuladores no confieren motivos para declarar lo sin imputables, ellos simulan esas patologías que el Código Penal acepta.

Para Ingenieros, todo delincuente es un individuo psicológicamente anormal, y esa anormalidad se origina en un "fondo degenerativo congénito o adquirido" (INGENIEROS, 1962a, p. 190). De modo que: "Todos los simuladores de la locura, por ser delincuentes, son mentalmente anormales; pero la posibilidad de simular la locura con fines jurídicos se da en razón inversa a esas anormalidades. Los delincuentes más degenerados son menos aptos para usar este medio defensivo en su lucha por la vida" (INGENIEROS, 1962a, p. 192). Al contrario, aquellos que tienen menos estigmas de degeneración tenderán a ser más simuladores.

Así, para establecer la correlación entre los delitos y las penas, resultaba necesario considerar un tercer elemento, un eslabón intermediario entre ambos: el sujeto delincuente. Foucault dirá que es con Garófalo (1885), y su 'ley del tercer elemento', que la figura del individuo peligroso ingresa en el sistema penal: "El derecho penal solo conocía dos términos, el delito y la penalidad. La nueva criminología reconoce tres, el delito, el delincuente y los medios de represión" (FOUCAULT, 2014, p. 249). Es en función de ese tercer elemento, un sujeto jurídicamente peligroso y psiquiátricamente degenerado, y no función del delito que debe definirse la pena.

Desde esta nueva perspectiva, los simuladores podrán dejar de representar un desafío epistemológico para el saber psiquiátrico. Ya no pondrán en evidencia sus límites, ni cuestionarán sus verdades, fingiendo síntomas y 
creando mentiras. Los delincuentes simuladores de Ingenieros, por presentar las anomalías propias de las diversas categorías de delincuentes, participan de la amplia galería patológica de los anormales y degenerados.

Cuanto más evidentes y notorios sean los estigmas de degeneración más difícil será que exista capacidad de crear estrategias de simulación que puedan engañar a los peritos: "Entre los delincuentes natos hay menos simuladores, no hay tendencia espontánea a la simulación" (INGENIEROS, 1962a, p. 102). Mientras que, entre aquellos anormales cuyos signos de degeneración son menos notorios, habrá mayor capacidad de crear estrategias de engańo, simulando las formas patológicas que pueden eximirlos de una pena. Se deduce de allí que: "La posibilidad de la simulación está en razón inversa al grado de degeneración psíquica del delincuente" (INGENIEROS, 1962a, p. 107).

En el derecho penal existente, habían fracasado todos los recursos empleados para desenmascarar a los simuladores: el uso de medios coercitivos, las drogas y tóxicos, los medios mecánicos como las duchas frías, o técnicas modernas como la plestimografia. Excluidas todas esas estrategias por ineficaces, Ingenieros concluye que

El único estudio específico de los delincuentes es, en nuestro concepto, el de su funcionamiento psíquico. Tienen las deficiencias comunes a los degenerados, pero de una manera especial; por eso no todos los degenerados encarrilan su actividad hacia la delincuencia. Estudiando la morfología criminal se observó en los delincuentes la existencia de los caracteres comunes a todos los degenerados. (INGENIEROS, 1962a, p. 184).

No todo degenerado es un delincuente, pero todo delincuente, sea o no simulador, es un degenerado. Así, la figura del delincuente simulador se transforma en una pieza clave para defender la modificación del Código Penal existente. Al proponer la substitución del criterio de responsabilidad por el de peligrosidad se abre la posibilidad de subordinar el derecho penal un discurso psiquiátrico que afirma ser capaz de identificar a los sujetos peligrosos ${ }^{15}$.

15 "Delinque porque tiene alguna deficiencia. El individuo peligroso se presenta como un sujeto enfermo" (GALFIORE, 2012, p. 3I). 


\section{Un dispositivo de seguridad}

Después de haber demostrado que la simulación de la locura por los delincuentes nace del criterio jurídico con que se aplica la pena, centrado en la responsabilidad, el objetivo de Ingenieros será anticipar la acción delictiva. Identificar al delincuente, incluso antes de que delinca. Pero, para crear un dispositivo de prevención y seguridad que permita prevenir delitos, parecía necesario resolver un viejo problema:

Un escollo encontrado es la falta de criterio uniforme para interpretar la alienación mental, separándola de la simulación. ¿Dónde termina la salud mental? ¿Dónde comienza la locura? Es una de las cuestiones más arduas presentadas al estudio de los alienistas, sin encontrarse una fórmula definitiva que solucione sus incógnitas. (INGENIEROS, 1962a, p. 175).

Si esa cuestión epistemológica parecía imposible de ser respondida era porque, de acuerdo a Ingenieros, se había desconsiderado la existencia de estados intermediarios entre normalidad y locura. Los últimos desarrollos de la psiquiatría heredera de Magnan y Legrain (1895) permitían reorganizar el derecho penal sobre nuevas bases científicas, en la medida en que permitían clasificar diversas anomalías psíquicas ${ }^{16}$. Por otra parte, y una vez "demostrado que la simulación de la locura por los delincuentes nace del criterio jurídico con que se aplica la pena, según la responsabilidad o irresponsabilidad del sujeto, la profilaxis debería consistir en una reforma jurídica que convierta (a la simulación) en nociva para el simulador" (INGENIEROS, 1962a, p. 175).

Siguiendo los postulados defendidos, desde 1890, por la Escuela Positiva de Antropología Criminal y por la Asociación Internacional de Derecho Penal, Ingenieros propone desplazar del eje de organización del sistema jurídico hacia el tema de la seguridad. "Reemplazando el criterio de irresponsabilidad del delincuente por la aplicación de la defensa social, proporcionalmente a su temibilidad, la simulación de la locura se tornará perjudicial para los simuladores, desapareciendo de la psicopatología forense" (INGENIEROS, 1962a, p. 103).

Las circunstancias jurídicas dejarán de favorecer a los simuladores cuando, sobre nuevas bases, el derecho penal se aproxime al desarrollo científico de

16 Para um análisis de los estados intermediários y las diferentes formas de degeneración en los criminales ver: Magnan, V E Legrain, P. Les Degénérés: État mental e Syndromes episodiques, Paris. Rueff, 1895, p. 206 e ss. 
la nueva psiquiatría. Entonces, "la simulación será desventajosa" y tenderá a desaparecer. Para que eso ocurra debía abandonarse la idea de que cabe a la justicia castigar la perversidad, las intenciones, la responsabilidad y la conciencia, pudiendo desconsiderar algo más relevante: la peligrosidad o temibilidad del delincuente (INGENIEROS, 1962a, p. 103).

Este desplazamiento operado en el campo médico-jurídico por Ingenieros, supone un nuevo modo de entender la relación entre criminalidad y enfermedad mental que ya estaba presente en el Capítulo VIII de Los degenerados de Magnan y Legrain (1895). Dirán que "por el hecho mismo de su estado mental, el degenerado se transforma, frequentemente, en delincuente" (1895, p. 181), tesis que Ingenieros retoma dando un paso más al afirmar, con Lombroso ${ }^{17}$, que todo delincuente es un degenerado. Magnan y Legrain defienden, como lo hará más tarde Ingenieros, la necesidad de modificar el viejo sistema penal dejando de lado el tema de la responsabilidad, substituyéndolo por la temibilidad. Concluyen, como lo hará Ingenieros, con la necesidad de garantizar la prevención y la profilaxis del crimen identificando la peligrosidad del sujeto. Esta última cuestión aparece no solo en la Simulación de la locura, sino también en el libro Criminologia, particularmente en el Capitulo VIII, denominado "Las nuevas bases de defensa social" 18 . Podemos decir que para Ingenieros, del mismo modo que para la psiquiatría analizada por Foucault:

[...] la noción de degeneración permitirá relacionar al menor de los criminales con un peligro patológico para la sociedad, para la especie humana en su conjunto. Todo el espacio de las infracciones podía concebirse en términos de peligro y por lo tanto de protección que tenía que ser asegurada. El derecho no tenía más opción que callarse o taparse las orejas y negarse a escuchar. (1981, p. 417).

Así, para defender la necesidad de una ruptura con el antiguo Código Penal, Ingenieros hará referencia al caso de un homicida simulador llamado Juan Passo (1903, p. 283). Esta persona fue considerada de manera diferente por dos jueces, que centraron sus evaluaciones en la responsabilidad. Un juez interpreta la responsabilidad tal como está en el Código Penal, afirmando

7 Sobre la crítica de Magnan y Legrain a Lombroso, ver: Les Degénérés, op cit p.192.

18 Extractos de este capitulo fueron publicados em 1914 en los Archivos de Criminologia. Psiquiatria y Medicina Legal bajo el nombre de: "Psiquiatria nueva y leyes viejas" (GALFIORE, 2012, p. 35). 
que Passo, no obstante ser un degenerado moral, no es un alienado y que es, en consecuencia, responsable por sus actos. El otro juez analiza el caso desde la perspectiva psiquiátrica, afirmando que existen abundantes indicios de degeneración y consecuentemente se trataría de un alienado, esto es, un sujeto irresponsable.

Ingenieros concluye que esa discrepancia no hubiera existido si en lugar de centrar el debate en el tema de la "responsabilidad", los jueces se hubieran preguntado por la "peligrosidad" del individuo: "Ambas partes coincidieron, en efecto, en señalar su intensa degeneración moral. Y para un degenerado moral, peligroso por la ausencia de sentimientos sociales, nadie se hubiera atrevido a pedir la absolución" (INGENIEROS, 1962b, p. 284). La misma estrategia es utilizada por Magnan e Legrain (1895). Analizan diversos actos criminales cometidos por diferentes tipos de degenerados, presentando dos perspectivas diferentes. Muestran que estos individuos pueden ser vistos como irresponsables por algunos y como responsables por otros, aunque cuando se evalúa el grado de peligrosidad aparecen las coincidencias. Igual que Ingenieros, frente a la pregunta por la responsabilidad o irresponsabilidad del criminal, Magnan y Legrain responden: "Nosotros no sabemos nada, ignoramos, no sabemos si él disfruta de su libre albedrío, solo sabemos una cosa: que amenaza los intereses de todos" (MAGNAN; LEGRAIND, 1895, p. 204).

Tal como la teoría de la degeneración lo había establecido, anormalidad psíquica y peligrosidad jurídica, caminan juntas: "Siendo ramas nacidas del tronco común de la degeneración fisiopsíquica, la criminalidad y la locura tienen estrecho parentesco. Así como el delito es más frecuente en los alienados que en los cuerdos, la locura se observa con más frecuencia en los delincuentes que entre los honestos" (INGENIEROS, 1962a, p. 185).

Ingenieros retoma también los postulados defendidos por la Asociación Internacional de Derecho Penal. Foucault resume esos postulados afirmando que: "La diferencia no estriba entre responsables que deben ser condenados e irresponsables que no deben serlo, sino entre sujetos absoluta y definitivamente peligrosos y aquellos que, mediante ciertos tratamientos, pueden dejar de serlo" (1981, p. 216).

En la alianza que se establece, a fines del siglo XIX, entre psiquiatría y discurso jurídico, entre degeneracionistas y escuela de antropología positiva, 
se trata de: abandonarla noción jurídica de responsabilidad y substituirla por la idea de temibilidad; mostrar que eran los individuos considerados irresponsables por el derecho penal, quien es representaban mayor peligro para la sociedad; rediseñar la función social del castigo y el carácter de la condena penal, transformándola en una estrategia preventiva y de defensa social (FOUCAULT, 1981, p. 412).

En ese marco general debemos situar la respuesta de Ingenieros al problema de la simulación. Ante la imposibilidad de identificar a los delincuentes simuladores, ante la relatividad y la falta de certeza de los medios entonces existentes para descubrir sus engaños, el único camino posible era crear estrategias de anticipación y disuasión. En sus palabras, si "no hay certeza absoluta de que el simulador no pueda engañar a los peritos, burlándose de la justicia", entonces, "solo puede suprimirse la simulación de la locura por los delincuentes, previéndola" (INGENIEROS, 1962a, p. 286). Por ese motivo Ingenieros se propone:

Reemplazar las bases absurdas del actual sistema punitivo, por las bases científicas de la psicopatología, que hagan más eficaz la defensa de la sociedad contra los actos delictuosos. De igual modo, la pena, degradada por la idea de venganza o de castigo a la que se la asocia, debe ceder su puesto a una más amplia y segura defensa social. (INGENIEROS, 1962a, p. 289).

Se trata de proponer un nuevo modo de articular los delitos y penas. Tomando como punto de partida las estrategias, tanto profilácticas como represivas de defensa de la sociedad, propuestas por Garófalo (1885), Lombroso (1892) y Ferri ${ }^{19}$ (1895), Ingenieros dirá que sigue el programa científico de defensa social establecido por la escuela positiva ${ }^{20}$. Este programa diferencia cuatro categorías punitivas: medios preventivos; medios

19 Para un estudio más detallado de los problemas que caracterizan a la antropología positiva, ver: Galfiore (20/2), Ferla (20/4), Campos (2000), entre otros.

20 Magnan y Legrain presentan algunas objeciones a la teoría de Lombroso desde el punto de vista de la teoría de la degeneración. Afirman que crimen y degeneración no pueden ser identificados en todos los casos, aunque existan degenerados que pueden devenir criminales, otros presentan estigmas de degeneración atenuados, y existen también delincuentes que no presentan estigmas de degeneración (1895, p. 192). Al analizar los casos de Ingenieros se observa una clara distinción entre criminales natos, caracterizados como fuertemente degenerados (que simulan menos), criminales que poseen menos estigmas de degeneración (los que más simulan) y otros que no poseen estigmas de degeneración. Como afirma Lakoff (2012, p. 856) existe en Ingenieros cierto "disagreement with Cesare Lombroso and the majority of European criminologists, for whom inherited degeneracy was the best way to explain criminality". 
reparadores (indemnización de la víctima); medios represivos (penas variables) y medios eliminadores (reclusión perpetua, deportación, y pena de muerte) (INGENIEROS, 1962a, p.290).

Sin embargo, para que estos medios de protección social no se limiten a repetir las antiguas evaluaciones jurídicas, centradas en el delito y en la teoría de la proporcionalidad de la pena, será necesario dar un paso más. La justicia deberá centrar su atención en los elementos antisociales que amenazan a la seguridad, desplazando el foco del delito a la personalidad del delincuente. Es así como Ingenieros describe este proceso:

Siendo su objeto impedir que el delincuente ejercite de nuevo sus tendencias antisociales, su consecuencia lógica es que la pena no debe adaptarse al delito, considerado abstractamente - sino al delincuente, considerado como una peligrosa realidad concreta [...]. La posición de los delincuentes cambia mucho si se funda la represión penal en su temibilidad y no en su responsabilidad. (INGENIEROS, 1962a, p. 290).

Con este desplazamiento se invertirá también el papel de los discursos jurídico y psiquiátrico. Si en el derecho penal clásico la decisión sobre la forma de la pena recaía en el ámbito de la justicia, ahora el discurso jurídico deberá subordinarse a la evaluación psicopatológica del delincuente. Cuando se trata de temibilidad, solo el saber psiquiátrico parece ser competente para evaluar el grado de peligrosidad que un delincuente representa o representará en el futuro. Es la psiquiatra quien debe definir en qué categoría de anormalidad un sujeto si inscribe, y definir el tipo de castigo apropiado.

Dando un paso más en la dirección establecida por Ferri (1895), enuncia tres formas de castigo o represión, una represión máxima destinada a los delincuentes natos, pero también a los habituales e incorregibles; una represión mediana, destinada a los delincuentes habituales corregibles, con psicosis transitoria; y una represión mínima para los criminales ocasionales e impulsivos accidentales.

En ese nuevo derecho penal centrado en la seguridad social y en la temeridad del delincuente, donde la justicia se subordina a la psiquiatría, es allí que, finalmente, Ingenieros enunciala única estrategia que considera viable para enfrentar el problema de la simulación. Ya no se trata de descubrir el engaño o la ficción, tarea que reconoce imposible, sino de anticiparse a la 
ocurrencia del fraude, esto es, garantizar "la profilaxis jurídica de la simulación de la locura” (INGENIEROS, 1962a, p. 256).

Resulta necesario, para Ingenieros, transformar la simulación de la locura en un acto perjudicial para los delincuentes simuladores, tan nocivo que haga desaparecer ese problema de la psicopatología forense. Para ello, las consideraciones jurídicas debe subordinarse a un estudio clínico-psiquiátrico de los delincuentes, centrado en la determinación del grado de peligrosidad (INGENIEROS, 1962a, p. 291). De ese modo se transformará el estatuto jurídico del simulador. La simulación dejará de ser una coartada eficaz y se transformará en un factor indicativo de represión penal agravada.

$\mathrm{El}$ argumento de Ingenieros se centra en mostrar que, si observamos la atribución de las penas considerando la temibilidad y no la responsabilidad en ningún caso puede resultar ventajoso para el delincuente simular la locura. En el caso de los alienados delincuentes diferencia dos tipos de pena, una máxima para los que poseen psicosis congénita o adquirida de manera permanente y una pena mediana para los que realizaron un delito como consecuencia de un trastorno transitorio. Dirá que la simulación, por definición, se refiere a estados permanentes que perduran después de cometido el acto, y que se mantienen a lo largo de la pericia, de ese modo, el delincuente simulador correrá el riesgo de recibir una pena máxima. Tampoco resultará conveniente simular locura para los delincuentes pasionales o de ocasión, porque por igual motivo, también se arriesgan a recibir una pena máxima. Los delincuentes natos, no obtendrían ventaja en simular porque a ellos de todas formas les correspondería, de acuerdo al criterio de peligrosidad, una pena máxima.

En este último caso, puede inclusive representar una desventaja, porque "la locura, agregada a la criminalidad por intensa degeneración implica una mayor temibilidad del sujeto" (INGENIEROS, 1962a, p. 292). Así, simplemente por utilizar como estrategia de disuasión la gravedad de la pena, Ingenieros deduce que la simulación tenderá a desaparecer. Los delincuentes descubrirán que esa ficción los perjudica penalmente, y que de ese modo se arriesgan a recibir una pena máxima. Podemos decir que, para Ingenieros, lo que permite resolver el problema de la simulación se resume en este aforismo de la psiquiatría forense: "La locura no es causa eximente de responsabilidad, sino agravante de la temibilidad del que la sufre, haciendo necesaria una mayor rigurosidad de la defensa social" (INGENIEROS, 1962a, p. 292). 


\section{Para concluir}

Podemos decir que la estrategia presentada por Ingenieros para erradicar el problema de la simulación no es otra que la disuasión. De modo que, más que dar una respuesta al problema de la simulación, todo parece indicar que Ingenieros sueña con su desaparición. Esa desaparición se logrará cuando la legislación penal se transforme, privilegiando la temibilidad sobre la responsabilidad. Entonces el delincuente renunciará a simular un estado de locura que pasará a ser considerado como agravante para la pena. Es el miedo, elevado a la categoría de profilaxis social, lo que permitirá anticipar y evitar las simulaciones. La respuesta de Ingenieros al problema de la simulación no significa otra cosa que la esperanza de que el delincuente renuncie a ficcionar su locura, por temor a un castigo que puede ser aún mayor de aquel que le correspondería por su crimen.

Si recordamos que el problema de la simulación es uno de los grandes temas de la psiquiatría desde Pinel, que autores como Laurent o el mismo Magnan intentaron crear estrategias para desenmascarar a los simuladores con el objetivo de proteger a los verdaderos alienados, no deja de ser inquietante la respuesta de Ingenieros. Lo que vemos allí es un reconocimiento explícito de la incapacidad del saber psiquiátrico para descubrir a los simuladores.

Ese reconocimiento deja al desnudo la dificultad, inherente a la psiquiatría, para establecer parámetros de diferenciación entre lo normal y lo patológico. Así, y ante la imposibilidad de dar respuesta al problema epistemológico planteado por los simuladores, esto es, al problema de la legitimidad, validez y límites del saber psiquiátrico, Ingenieros recurrirá, al ejercicio del poder psiquiátrico: represión máxima para los delincuentes que posean indicios de temibilidad y castigo ejemplar para los anormales o degenerados que han cometido un delito. Ya no importa el delito cometido, sino el riesgo que el sujeto representa para la sociedad.

La transformación del Código Penal, abandonando la responsabilidad y substituyéndola por la temibilidad, permitiría no solo anticipar y evitar las ficciones creadas por los simuladores ante el temor de una punición agravada, sino también, terminar con el desafío epistemológico que ellos representaban. Pues, lo que los simuladores ponían en cuestión no era otra cosa que las certezas sobre las cuales se fundaba la psiquiatría, su capacidad de determinar si un individuo era o no loco, si era o no inimputable. 
Es evidente la persistencia de los problemas analizados por Ingenieros en 1903 a lo largo del tiempo. Hoy observamos, como a inicios del siglo XX, una proliferación de diagnósticos psiquiátricos ambiguos para comportamientos socialmente indeseables. Las imprecisas y epistemológicamente frágiles categorías psiquiátricas definidas por el DSM-III (APA, 1980) y sus versiones posteriores, parecen multiplicarse al infinito como forma de anticipar posibles situaciones de violencia, delito, homicidio o suicidio. El continuo psiquiátricocriminológico que se estableció en tiempos de Ingenieros permanece hasta hoy, cuando se insiste en la "temibilidad" y la seguridad como forma de anticipación de delitos. Es así que nuestras cárceles pueden estar repletas de individuos sin claras evidencias de crímenes cometidos, pues lo que se juzga no es el acto sino la supuesta peligrosidad de esos sujetos. De igual modo, los diagnósticos psiquiátricos se multiplican fundamentalmente en la infancia y la adolescencia, legitimándose en la idea de prevención del delito, al mismo tiempo que se multiplica e naturaliza el uso de terapéuticas farmacológicas de dudosa eficacia.

\section{Referencias}

AMERICAN PSYCHIATRIC ASSOCIATION Diagnostic and Statistical Manual of Mental Disorders, Fifth Edition (DSM-III). Arlington: Ed. American Psychiatric Association, 1980.

BIAGINI, H. El movimiento positivista argentino. Buenos Aires: Ed. De Belgrano, 1980

CAMPOS MARÍN, R. Los Ilegales de la naturaleza, medicina y degeneracionismo en la España de la restauración. Madrid: CSIC, 2000.

COFFIN, J. La transmission de la folie- 1850-1914. Paris: ed. Harmattan. 2003.

CAPON, S. Locos y degenerados: una genealogia de la spiquiatria ampliada. Buenos Aires: Ed Lugar, 2014

FALCONE, R. Genealogia de la locura: discursos y prácticas de la alienación mental en el positivismo argentino 1880-1930, Buenos Aires: Ed. Letra Viva, 2012.

FERLA, L. La pericia médico legal como forma de producir la Verdad y sus adversarios en los tribunales brasileños de Entreguerras. Asclepio - Revista de Historia de la Medicina y de la Ciencia, v. 66, n. 2, p. 1- 13, 2014. 
FERNANDEZ, C. B. ¿'Teorías científicas fuera de lugar?. Algunas derivas del evolucionismo en el positivismo argentino. Hispanic Research Journal, v. 7, n. 3, p. 223-236, 2006.

FERRI, E. Socialismo y ciencia positiva. Buenos Aires: Imprenta de La Nación. 1895.

FOUCAULT, M. Les Anormaux. Paris: Gallimard, 1999.

L'evolution de la notion d'individu dangereux dans la psychiatrie legale. Déviance et Société, v. 5, n. 4, p. 403-422, 1981.

Le Pouvoir Psiquiatrique. Paris: Gallimard, 2003.

Securité, Territoire, population. Paris: Gallimard, 2004.

Obrar Mal, decir la verdad. Buenos Aires: Siglo XXI, 2014.

GARBINI, G. La simulation de la folie. A, In Annales médico-psychologiques. Paris: n. 3, $1906 n^{\circ} 03.1906$.

GALFIORE, M. C. La sociología criminal de Enrico Ferri: entre el socialismo y la intervención disciplinaria. In: VII JORNADAS DE SOCIOLOGÍA DE LA UNIVERSIDAD NACIONAL DE LA PLATA, 7., La Plata, 2012. Disponible en: <http://jornadassociologia. fahce.unlp.edu.ar>. Acceso en: 2017.

GARÓFALO, R. La Criminología. Estudio sobre el delito y la teoría de la represión. México: PDM, 1885.

HUERTAS, R. Locura y Degeneración. Cuadernos Galileo de História de las Ciencia 5. Madrid: Ed. Centro de Estudios Históricos, 1987.

INGENIEROS, J. Simulación de la locura. Obras Completas. 1. ed. 1903. Tomo 1. Buenos Aires: Ed. Mar Oceano, 1962a.

. [1916]. Criminologia. Obras Completas. 1. ed. 1916. Tomo 1. Buenos Aires: Ed. Mar Oceano, 1962b.

[1919]. La locura en Argentina. Obras Completas. 1. ed. 1919. Tomo 1. Buenos Aires: Ed. Mar Oceano, 1962b.

LAKOFF, A. The Simulation of Madness: Buenos Aires, 1903. Critical Inquiry, v. 31, n. 4, p. 848-873, 2005.

LAURENT, A. Étude médico-légale sur la simulation de la folie. Paris: Victor Masson et fils. 1866 . 
LEGRAIN, P. M. Dégénérescénce sociale et alcoolisme physiques. Annales d'hygiéne publique et de médecine legal, n. 34, 1895.

LOMBROSO, C. Les applications de l'anthropologie criminell. Paris: Felix Alcan Editeur, 1892.

MAGNAN, V. Leçons cliniques sur les maladies mentale. Paris: Baillére, 1887.

.; LEGRAIN, P. M. Les dégénérés: état mental et syndromes épisodiques. Paris: Baillére, 1895.

MAGNAN, V. Recherches sur les centres nerveux. Alcoolisme, folie des héréditaires dégénérés. Paris: Masson, 1893.

MIRANDA, M.; VALLEJO, G. (Org.). Uma Historia de la Eugenesia. Argentina y las redes biopoliticas internacionales 1912-194. Buenos Aires: Biblos, 2012.

MOLLOY, S. The politics of posing. In: CONSTABLE, L. et al. (Ed). Perennial Decay: on the aesthetics and politics of decadence. Philadelphia: University of Pennsylvania Press, 1988.

MOREL, B. A. Rapport Medical sur un cas de simulation de folie. Annales médicopsychologiques, n. 3, p. 61-83, 1857.

PICK, D. Faces of degeneration. A european disorder 1848-1918. New York: Cambridge University Press, 1999.

PINEL, P. Traité Médico- Philosophique sur l'aliénation mentale. Paris: Les empecheurs de penser en rond. (1809), 2005.

SIMENON, G. Maigret hésite. Paris: Le Livre de Poche, 1968.

STAGNANO, J. C. Evolución y Situación de la Historiografía de la psiquiatría Argentina. Rev. Frenia, v. 1, p. 7-37, 2006.

TRUELLE, V. La obra Médico legal de Magnan. Annales médico-psychologiques, n. 1, p. $760-771,1935$.

TERÁN, O. Positivismo y nación en la Argentina. Buenos Aires: Ed puntosur, 1987.

VERMEREN, P.; VILLAVICENCIO, S. Positivismo y ciudadanía: José Ingenieros y la constitución de la ciudadanía por la ciencia y la educación en la Argentina - CUYO. Anuario de Filosofía Argentina y Americana, n. 15, p. 61-78, 1998.

VEZZETTI, H. El nacimiento de la psicología en Argentina. Buenos Aires: Ed Puntosur, 1988. 


\section{Degenerate and Simulators: a foucauldian reading of "The Simulation of Madness” by José Ingenieros}

\section{Abstract}

This article discusses the problem which is the axis of the study that José Ingenieros dedicated to the simulation of madness: the simulating criminals. These are individuals who have committed a crime and that, in order to break free from prison, pretend to have acted without awareness of their actions. By mediating the simulators figure, Ingenieros shows the precise moment that begins, in Argentina psychiatry, the building of that psychiatric-criminological continuum that allows address the most varied legal issues from a medical point of view. From that moment, it will be possible, through the mediation of the theory of degeneration, that an undefined set of behaviors enter in the field of psychiatric observation. For Ingenieros, Criminal Law only could resolve the problem of simulation by integrating that new psychiatric perspective.

Keywords: José Ingenieros. Degeneration. Law. Psychiatry. Simulation.

Recebido em: 14/12/2016

Aceito em: 22/02/2017 